PO 8382 CLINICAL AND LABORATORY ASSESSMENT OF LOA LOA INFECTIONS IN GABON

${ }^{1}$ Luzia Veletzky, ${ }^{2}$ Manego Rella Zoleko, ${ }^{2}$ Daniel Stelzl, ${ }^{2}$ Jennifer Hergeth, ${ }^{2}$ Lia B Dimessa Mbaginga, ${ }^{3}$ Christine Budke, ${ }^{1}$ Johannes Mischlinger, ${ }^{2}$ Ghyslain Mombo-Ngoma, ${ }^{2}$ Ayola Akim Adegnika, ${ }^{4}$ Wolfram Metzger, ${ }^{5}$ Heimo Lagler, ${ }^{4}$ Peter $G$ Kremsner, ${ }^{4}$ Benjamin Mordmüller, ${ }^{1}$ Michael Ramharter. ${ }^{1}$ Bernhard-Nocht-Institute for Tropical Medicine and University Medical Center Hamburg-Eppendorf, Germany; ${ }^{2}$ Centre de Recherches Médicales de Lambaréné, Libreville, Gabon; ${ }^{3} T e x a s$ AandM University, Bryan, Texas, USA; ${ }^{4}$ Institute for Tropical Medicine University of Tuebingen, Germany; ${ }^{5}$ Department of Medicine I, Division of Infectious Diseases and Tropical Medicine, Medical University of Vienna, Austria

\subsection{6/bmjgh-2019-EDC.79}

Background Loiasis is a parasitic infection highly endemic in West and Central Africa. Previously often considered as a benign infection, recent studies have suggested that high microfilaria loads in loiasis patients may be associated with increased mortality. The true impact of loiasis on affected communities is unknown. Reports on clinical symptoms and changes in laboratory values due to loiasis infection are focused mostly on returning travellers. Assessments in endemic populations have scarcely been performed. Laboratory alterations such as eosinophilia are thought to be present mostly in patients from non-endemic areas.

Methods This cross-sectional study was performed in rural Gabon to investigate the clinical symptoms and laboratory changes caused by loiasis in an endemic population. Inclusion criteria were local residency for at least two years and an age above two years. Participants were interviewed with a questionnaire covering loiasis-specific symptoms, exploratory questions and history of eye worm. Local vocabulary was employed if needed. At the same time L. loa microfilaria diagnostics were performed including thick blood smear preparation and concentration techniques. Additionally, differential blood count and haemoglobin measurements were performed. Peripheral blood withdrawals were done between 10 am and 3 pm.

Results A total of 1030 participants were recruited, of whom $56 \%$ were female; they were between 2 and 98 years of age. L. loa microfilariae were detectable in $27 \%$ of all participants. Microfilariae densities ranged from 1 to $76250 \mathrm{Mf} / \mathrm{mL}$. Overall a positive history of eye worm was reported in $56 \%$, with $25 \%$ of participants reporting eye worm passage and $36 \%$ Calabar swelling within the last year. Also, an analysis of laboratory parameters in comparison to clinical data and infection status was performed.

Conclusion Loiasis infection should receive more attention by the scientific community and further studies are needed, as the disease causes substantial morbidity in endemic populations.

\section{PO 8383 THE ROLE OF PLASMA B CELLS IN MYCOBACTERIUM TUBERCULOSIS INFECTION AND DISEASE}

Awa Gindeh*, Simon Donkor, Olumuyiwa Owolabi. Medical Research Council, Gambia Unit, Serrekunda, The Gambia

\subsection{6/bmjgh-2019-EDC.80}

Background Tuberculosis (TB) is still a major global health problem with about one-quarter of the global population infected with the causative pathogen, Mycobacterium tuberculosis $(\mathrm{Mtb})$. The role of $\mathrm{T}$-cells in the adaptive immune response against Mtb has been extensively studied with little information on the role of B-cells. B-cells produce antibodies and differentiate into plasma and memory B-cells. Plasmablasts are a subset of plasma cells only present in the peripheral circulation following an ongoing infection or vaccination. Immunoglobulin G (IgG) especially IgG2 mounts more efficient immune response against bacterial infections, mainly attributed to the high affinity of IgG2 binding to the $\mathrm{Fc} \gamma$ receptor. Therefore, we hypothesised that Mtb-specific IgG + plasmablasts may be a useful biomarker of $\mathrm{TB}$ infection status.

Methods Ex-vivo B-cell enzyme-linked immunospot (ELISPOT) was used to identify plasmablasts responses to Mtb-specific antigens ESAT-6/CFP-10 (EC), together with non-specific Mtb purified protein derivative (PPD) and a positive (total $\mathrm{IgG}$ ) and negative (media only) control from adults with active TB pre- and post-treatment $(n=20)$ or with latent TB infection (LTBI; $\mathrm{n}=20$ ) in The Gambia.

Results Frequencies of Mtb-specific plasmablasts were significantly higher in active TB cases pre-treatment compared to post-treatment $(\mathrm{p}<0.0001)$ and LTBI with no difference seen following PPD stimulation. Interestingly, total IgG +cells were lower in the cases at recruitment but increased following treatment indicating the relative proportion of Mtb-specific responses were also significantly different $(p=0.034)$ prior to therapy.

Conclusion These data show that B-cell responses are differentially modulated during active and latent TB infection, suggesting that plasmablasts may be a useful biomarker for $\mathrm{TB}$ infection in TB-endemic settings.

\section{PO 8388 THE GLOBAL HEALTH TRAINING CENTRE PROFESSIONAL DEVELOPMENT SCHEME: CAPACITY DEVELOPMENT AND CAREER TRACKING FOR RESEARCHERS}

Elizabeth Rapa. University of Oxford, UK

\subsection{6/bmjgh-2019-EDC.81}

Background Health researchers need a free, online and reliable mechanism to record their skill set, track their career development, and identify gaps in their knowledge. Research teams also require ways to monitor and report their team's capacity development over time. The Global Health Network, a trusted and respected digital platform, is now able to provide researchers with these tools (www.globalhealthtrainingcentre.org/pds).

Methods The Global Health Network compared diverse data types and took into account user feedback to create a professional development scheme (PDS). Together with TDR (The Special Programme for Research and Training in Tropical Diseases), a framework was developed with a list of the core competencies that a research team needs to demonstrate to carry out a study succesfully. A multi-functional dashboard was designed for team coordinators to assess the capacity development of individuals and groups.

Results The PDS provides researchers with a step by step process for recording their career and core competencies. The core competency framework can be applied to any research study, regardless of size of the team, place, disease focus or type of research. Team coordinators can quickly and easily produce visual records of the strengths and weaknesses in the competencies of their team and implement plans to address these gaps. Together with supporting tools in the PDS, research teams can plan staffing requirements for a study, 